\title{
The Role of Airborne LiDAR Survey Technology in Digital Transformation
}

\author{
Trudy R Ganendra ${ }^{1, *}$, and Ebrahim Taherzadeh Mobarakeh ${ }^{1}$ \\ ${ }^{1}$ Ground Data Solutions R\&D Sdn Bhd, Lot 6, Jalan 51A/223, 46100 Petaling Jaya, Selangor, \\ Malaysia
}

\begin{abstract}
Civil engineering includes a wide range of applications that require three-dimensional (3D) information. With the advent of LiDAR (Light Detection And Ranging) technology, obtaining 3D terrain information accurately in a cost and time effective manner became more popular. In this paper, three case studies demonstrate the roles and applications of airborne LiDAR data including highway, transmission line and hydropower dam development projects. In first case study, the highway alignment was considerably changed after the initial design stage. LiDAR data was able to do the re-alignment survey quickly, allowing for prompt changes in design. In the next case study, a comparison is made between conventional transmission route alignment design and the design using PLS-CADD with LiDAR data. Utilizing the LiDAR data in PLSCADD allows transmission line engineers to model the construction costs of several alignments options effectively and efficiently compared to the conventional method. The last case study uses LiDAR for obtaining the necessary information for proposed dam. Using LiDAR data maximizes topological accuracy and volume measurements while minimizing survey time and costs. Furthermore, utilizing the multi-temporal LiDAR data can reveal valuable information such as topography changes. LiDAR survey technology has proven that it is the best method to capture accurate data from the earth surface which provides maximum density of topographical $\&$ digital imagery information for various applications.
\end{abstract}

\section{Introduction}

Analogue data is data that is represented in a physical way, which has some limitations in terms of collecting, storing, accessing, processing, sharing and visualizing this type of data. Civil engineering demands fluency with a great deal of information, and much of this information is traditionally analogue. With the advent of digital data we are able to overcome these limitations.

LiDAR, which stands for Light Detection and Ranging, is a surveying method that measures the distance to a target by illuminating the target with pulsed laser light, measuring the reflected pulses with a sensor, and calculating it from the time taken. This

\footnotetext{
* Corresponding author: admin@grounddatasolutions.com
} 
can then be used to make digital 3-D representations of the target. One inherent feature of LiDAR data is acquired, processed, and delivered digitally. The ability of LiDAR data to provide maximum accuracy and density of topographical \& digital imagery data while minimizing survey time and cost has led to various industries to utilize this technology for their various applications, including engineering, infrastructure, environment, disaster, and natural resources. Some of the applications of LiDAR data in civil engineering are as detailed below:

a) Pipeline, railway and road surveying: LiDAR helps in design, feasibility studies, slope stability monitoring, and maintenance of pipelines, railways and roads. LiDAR also provides accurate information for slope stability analysis, access facilitation at all points of routes, efficient planning and environmental impact analysis, as well as assisting in railway, road and pipeline risk management, which helps assure safety in the design, construction, operation, maintenance, and emergency response capacity of these facilities.

b) Flood Mapping: LiDAR helps in flood management [1], prediction, emergency response planning, evacuation planning, and mitigation work by providing designs and precise drainage studies of surface water. LiDAR also allows for inventorying of wetlands and inspection of water quality.

c) Telecommunications and urban planning: LiDAR allows for accurate 3D-modeling from high density digital data for use in telecommunications and urban planning [2] it assists in optimizing telecommunication networks, transportation planning, management and design. In addition, LiDAR helps with analysis and planning of vegetation cover in residential zones, parks, and recreation areas by providing views of proposed works in existing landscapes.

d) Transmission line surveying: LiDAR helps in new transmission lines design, existing transmission line maintenance (including vegetation and other encroachment management), line re-rating and line upgrading $[3,4]$. LiDAR technology is able to map all obstructions in the catenaries of existing transmission lines, which are crucial elements in tower spotting. LiDAR data is fully digital and highly adaptable for different uses, formats, transformation model, and software. LiDAR provides the ability to investigate project cost, construction time, earthworks, environmental impacts, and social impacts in a short period of time.

e) Catchments survey: LiDAR helps in water catchment feasibility studies, design and management [5], including degradation and monitoring due to erosion or forest clearing. LiDAR provides information to support the management of infrastructure (road, stormwater and wastewater) as well as spatial information that reflects the general state of the environment (including urban infrastructure, upper catchments, flood and coastal information), including land use mapping.

f) Dam survey: LiDAR helps in design, maintenance, feasibility studies, sedimentation studies and emergency planning. LiDAR maximizes topological accuracy and volume measurements while minimizing survey time and costs. It helps in optimizing dam infrastructure design and allows for slope stability analysis.

In this paper three case studies will demonstrate the roles and applications of airborne LiDAR data for highway, transmission lines and hydropower dam development projects.

\section{Methodologies}

\subsection{Case study: highway alignment}

Accurate terrain mapping is one of the important requirements for highway alignment planning and design, environmental impact assessment and infrastructure asset management $[6,7]$. 
The airborne LiDAR survey for East Coast Expressway (ECE) Phase 2 project, which is located on the East coast of Peninsular Malaysia is shown in Fig. 1. The corridor is approximately $200.5 \mathrm{~km}$ long and $1.5 \mathrm{~km}$ wide.

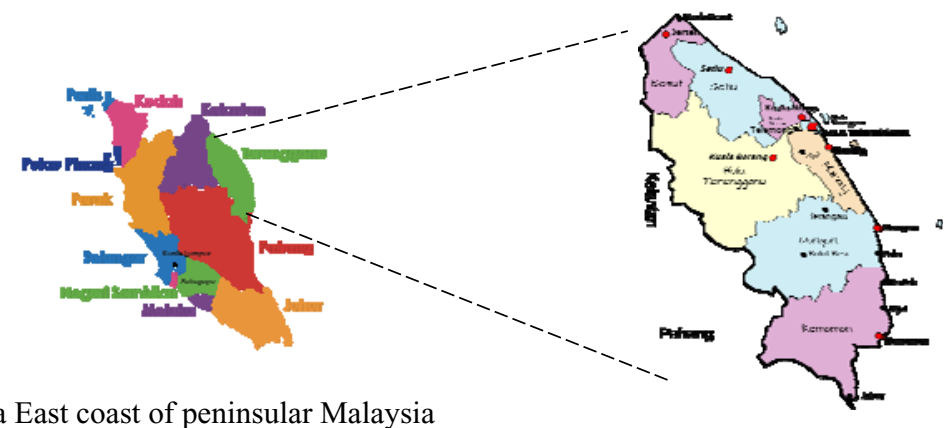

Fig. 1. Study area East coast of peninsular Malaysia

The LiDAR data was acquired within 22 Days (from 23 August 2004 to 14 September 2004). LiDAR point clouds was acquired with the point density 2 points $/ \mathrm{m}^{2}$. The Digital Elevation Model (DTM) and Digital Elevation Model (DEM) were delivered 7 days after the airborne acquisition. In 2005 the ECE alignment was changed due to changing social requirements and to facilitate the service to local communities. Thus, another airborne LiDAR survey was carried out based on the re-aligned corridor. The re-aligned corridor was from Ceneh to Bukit Besi and $105 \mathrm{Km}$ length and $1.5 \mathrm{Km}$ wide. The acquisition was completed in 10days and the DTM and DEM delivered a week after data acquisition. The area consists of various terrain conditions including oil palm plantation, rubber plantation, dense forest, open areas, urban areas, flat and undulating.

The main objective of these two survey projects was to produce the topographical and features map of the areas using airborne LiDAR technology for input into MOSS (MX ROAD) design software that enables the rapid and accurate design for road.

The special in-house program was used to process the raw LiDAR data (combined the data from the LiDAR sensor, GPS and IMU). TerraSolid software was used to classify and process the LiDAR data. The contour line was generated with 1 meter interval based on the finalized ground points. Some samples of products including LiDAR point cloud, 3D surface model and Ortho-rectified image overlay with 1 meter contour are shown in Fig. 2a, Fig. $2 \mathrm{~b}$ and Fig. 2c.

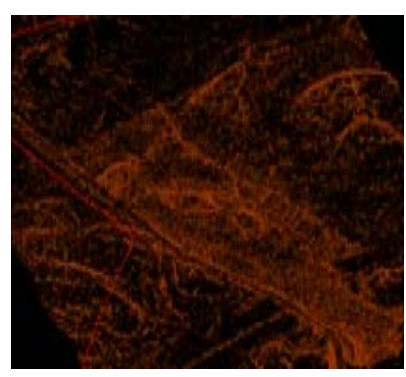

a)

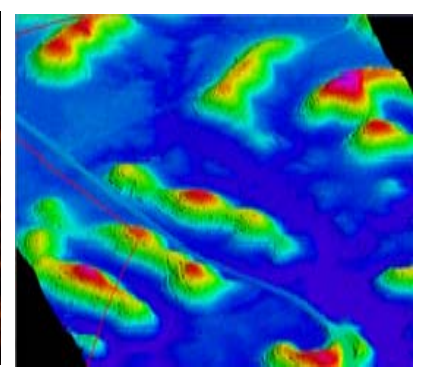

b)

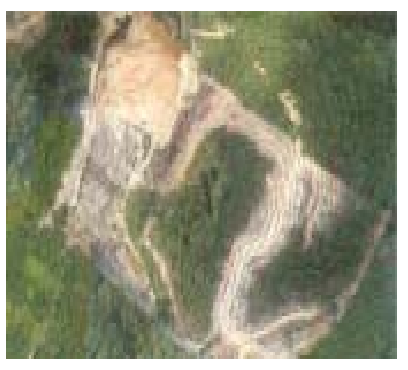

c)

Fig. 2. a) Lidar points of proposed Bukit Besi interchange b) 3D surface model of proposed Bukit Besi interchange c) contour overlay with orthophoto

The features relevant to the alignment planning and design were digitized using the orthorectified images. The digitized features and the contours were converted to MOSS format for the earthworks calculation and further analysis of the road alignments. In the fig 3. sample output from Moss software is presented. 


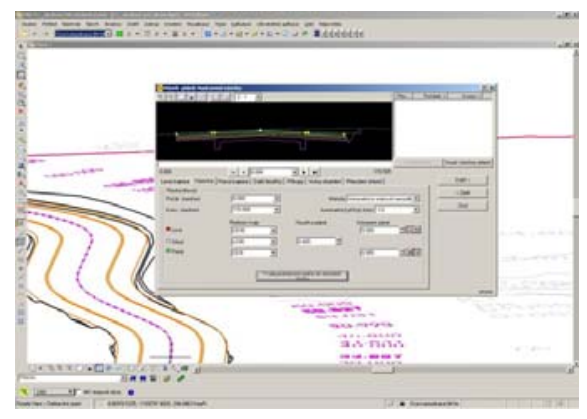

Fig. 3. Sample Output from Moss Software MOSS (MX Road) Software

An analysis of elevation data from LiDAR and Land Survey data for a sample area in the project showed high agreement [8]. The deviation of the data was between $0.06 \mathrm{~m}$ to $0.233 \mathrm{~m}$ at $95 \%$ confidence level. LiDAR and MOSS software were able to do re-alignment survey so quickly and allow for prompt changes in design such that there was little change to project schedule when more than $50 \%$ of the alignment was changed one year after initial survey was completed.

\subsection{Case study: transmission lines}

The study area consisted of two lines:

a) The $51 \mathrm{~km}$ of $275 \mathrm{kV}$ double circuit transmission line connecting Ulu Jelai main intake substation at Ulu Jelai renewable hydropower plant and Tapah main intake substation, and

b) $140 \mathrm{~km}$ of $500 \mathrm{kV}$ transmission line connecting Tapah substation to Bentong (South) substation for future interlink of HVDC system from Sarawak to Peninsular Malaysia (Bentong).

The total study areas corridor was approximately $190 \mathrm{~km}$ long and $0.5 \mathrm{~km}$ wide. The purpose of this survey was to provide all the necessary data and information required for a proposed $500 \mathrm{~m}$ wide route, enabling tower spotting using PLS-CADD and providing for a more optimized design.

The LiDAR data acquisition was carried out in 14 days. The collected LiDAR data had a point density greater than 4 points per $\mathrm{m}^{2}$, which is far higher when compared to conventional survey cross sections at $50 \mathrm{~m}$ intervals. The DTM, DEM, and contour were delivered 11 days after the airborne acquisition, and the final mapping was delivered in 35 days. The cross section of building in the study area and the LiDAR data of the existing transmission line wires are shown in Fig. 4a and Fig. 4b. 


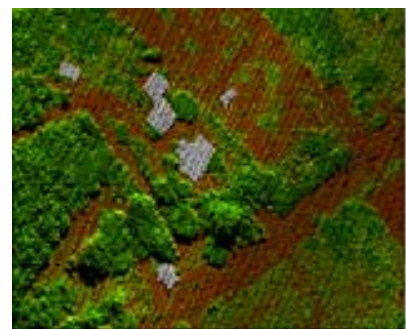

a)

(1)

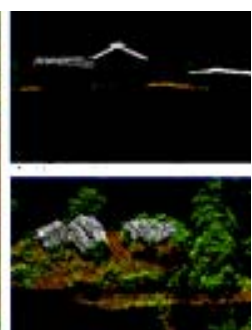

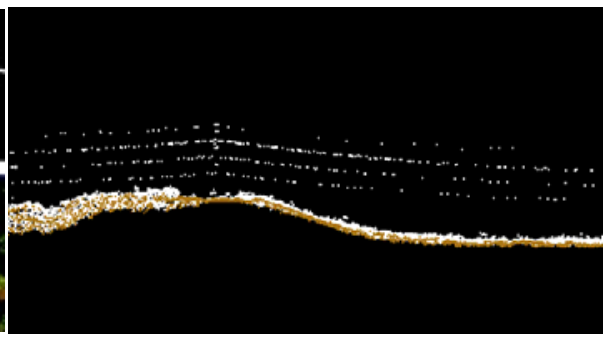

b)

Fig. 4. a) Cross section of buildings in the study area b) LiDAR data of the existing transmission line wires

TerraSolid and AutoCAD were used to conduct the data processing and feature digitizing. First, the data from the laser, IMU and GPS sensors were processed to produce a raw point cloud. Then, the points were classified into ground and non-ground points [9]. This included detailed ground editing to correct the errors in the automatic classification processes in order to improve the ground model. Then, the ground points were used to generate a DTM. The sample of digitized features with contours and the orthophoto are shown in Fig. 5a and Fig. 5b.

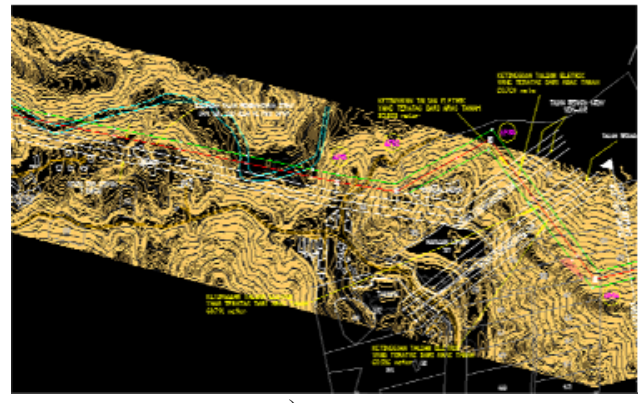

a)

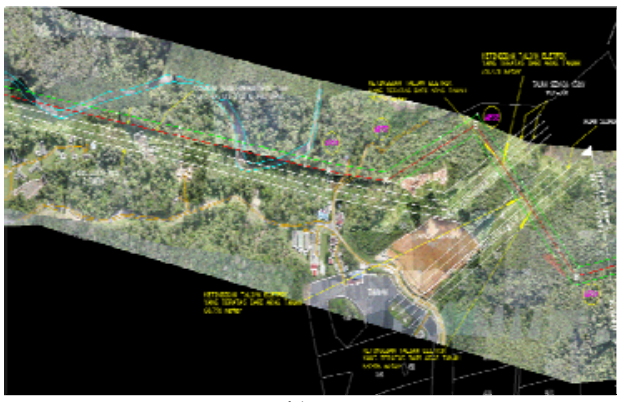

b)

Fig. 5. Sample of digitized features with a) contours b) orthophoto

LiDAR data can be imported and exported by most commercially available 3D software packages, including PLS-CADD. It is the premier overhead power line design software for the industry, which allows for fast, accurate, cost effective transmission line design. In this study, LiDAR is used as the primary source of data in PLS-CADD. Fig. 6 demonstrates the profile plan using PLS-CADD.
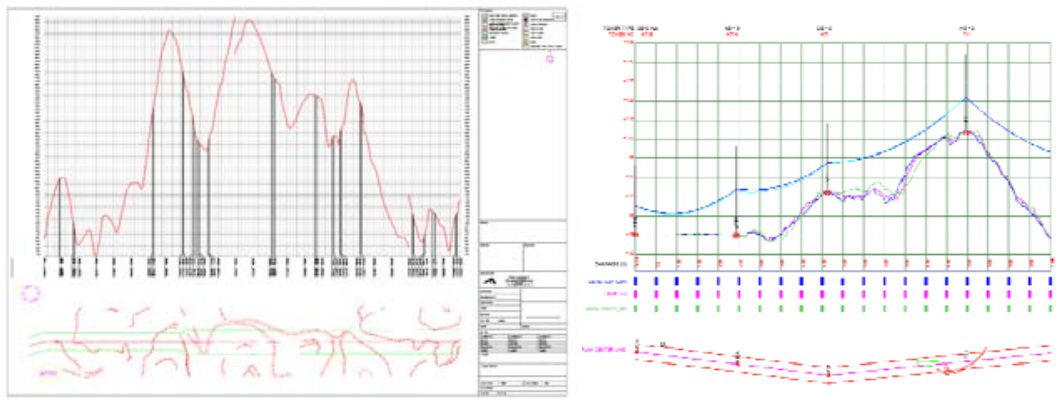

Fig. 6. The profile plan using PLS-CADD

A comparison was made between conventional transmission route alignment designs and the design using PLS-CADD with LiDAR data. Some examples of problems which 
occurred due to conventional design methodology accuracy and sparse data is detailed below:

1) River crossing: the location of AP11 selected by conventional design was found to be within river during the ground survey works. (Fig.7)

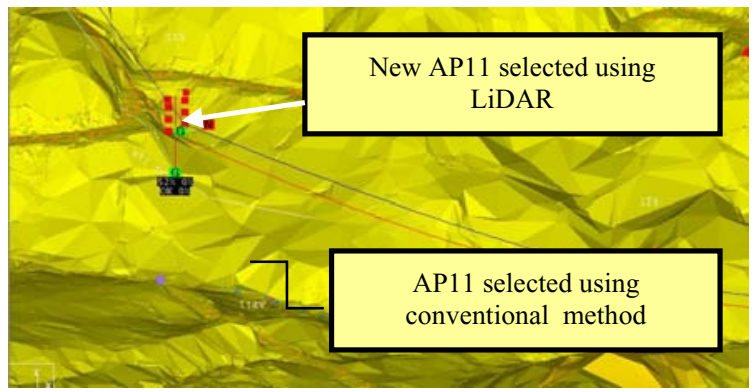

Fig. 7. Comparison of conventional methods and LiDAR survey when identifying the location of transmission lines

2) Identification of crossings: conventional methods cannot identify the best location for angle points where the proposed alignment crosses existing transmission lines. (Fig 8.)

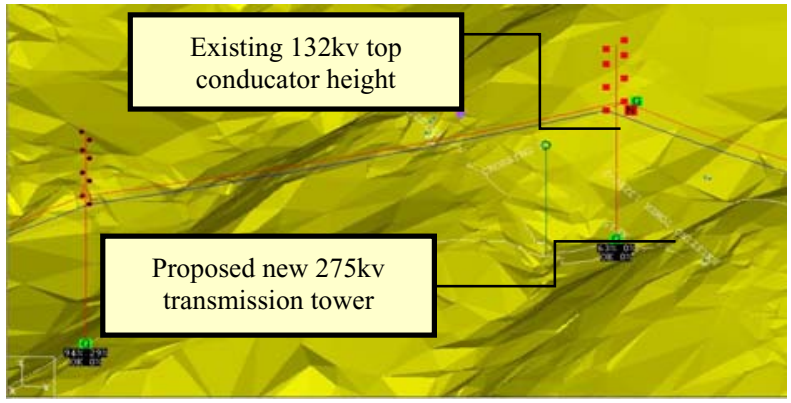

Fig. 8. Comparison between conventional methods and LiDAR surveys for angle point identification

3) Hilly terrain: work from the conventional method placed AP's in locations needing special towers, which are very expensive and difficult to construct (Fig 9.)

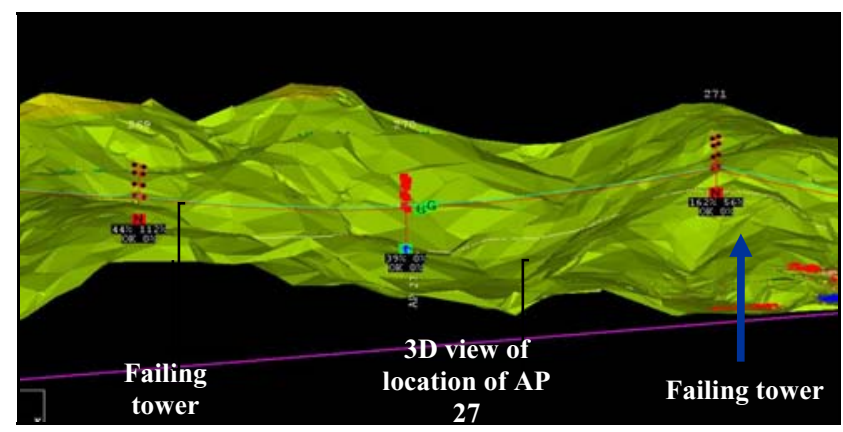

Fig. 9. Tower spotting in hilly terrain using conventional methods

LiDAR data allows transmission line engineers to model the construction costs of several alignments options effectively and efficiently. This study shows that the conventional method cannot identify the best location for angle point where the proposed alignment crosses existing transmission lines. In addition, LiDAR has the ability to identify existing transmission lines and other crossings which are crucial elements in tower spotting, 
which reduces project cost and time. Furthermore, LiDAR enables users to view the terrain in $3 \mathrm{D}$, allowing quick and easy tower spotting with quick repositioning of angle points and selection of suitable routes.

For this project, the final route selected would therefore have minimized deviations, construction costs and time. It was found that $29 \%$ of the proposed route derived from conventional transmission line route design required deviation, but utilizing LiDAR data and PLS-CADD could have prevented this.

\subsection{Case study: proposed hydropower dam development project}

The project area was located in Sarawak with total area approximately $4028.884 \mathrm{~km}^{2}$ (Fig 10). The purpose of this survey was to provide all the necessary data and information required for the ongoing studies, investigations and to design a number of potential hydropower development sites as a part of the Sarawak Corridor of Renewable Energy (SCORE) program. LiDAR contour mapping and aerial photography is an important component of the project.

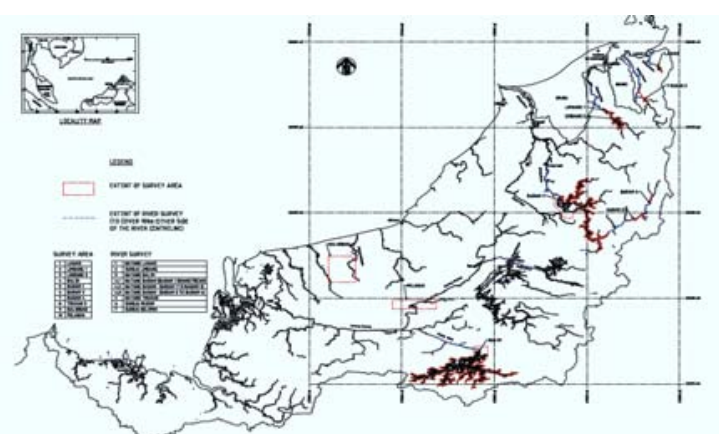

Fig. 10. Study areas

The LiDAR data was acquired within 25 weeks by involving 289 aerial flight missions. LiDAR point clouds were acquired with the point density between 2.2 and 10.68 points $/ \mathrm{m}^{2}$. The DTM and DEM were delivered 14 weeks after the airborne acquisition. Despite the unusual extremely heavy rainfall (106 rain-days out of 229 total acquisition days) during the annual dry season, this project was successfully performed and met or exceeded the required specifications per project Terms of Reference (TOR). TerraSolid and AutoCAD were used to conduct the data processing and feature digitizing. The ground point was used to generate the DTM and contour. The LiDAR point cloud and cross section over the building and vegetated area is demonstrated on Fig. 11a. The digitized features and contours overlaid with orthophoto is shown in Fig. $11 \mathrm{~b}$.

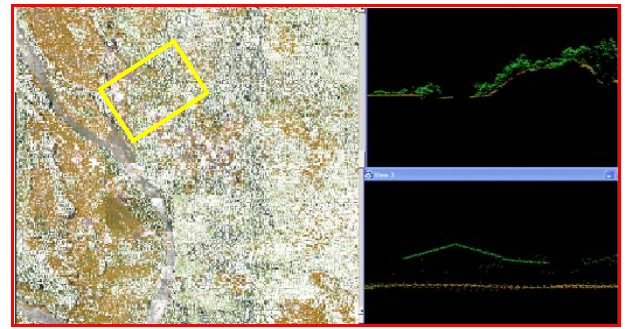

a)

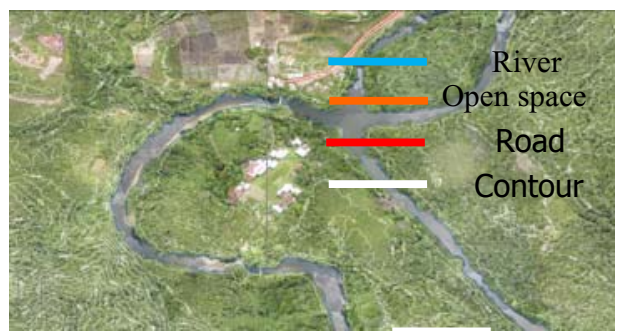

b)

Fig. 11. a) LiDAR point cloud and cross section view b) Digitized features and contours overlaid with orthophoto. 
LiDAR was seen to capture dam inundation area more accurately than conservative estimates using topographical map. LiDAR data showed many areas that were presumed outside the dam inundation area, would be flooded, leading to re-evaluation of land compensation issues. In Figure 12a, the original survey boundary derived from JUPEM topographical map which estimated the dam inundation area is shown. The Fig. 12b is shown the actual inundation area captured by LiDAR.

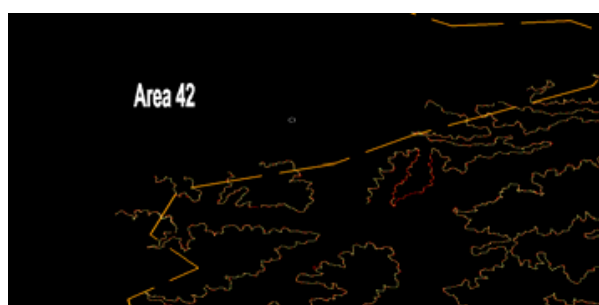

a)

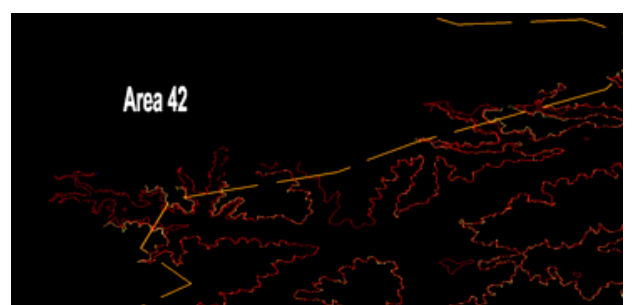

b)

Fig. 12. a) Original survey boundary derived from JUPEM topographical map which estimated the dam inundation area $b$ ) The actual inundation area captured by LiDAR

Soil volume calculations were performed using Terra Modeler, via the "compute quantity" tool. Three samples were taken from three different locations within the project area, and the volume calculation was performed using terra modeler and manually (Table. 1). The difference in the volumes calculated were $0.087 \%, 0.162 \%$, and $0.015 \%$ each, indicating that the terra modeler calculation is reliable and definitely much faster.

Table 1. Volume calculation

\begin{tabular}{|c|c|c|c|}
\hline Software & Sample $1\left(\mathrm{~m}^{3}\right)$ & Sample $2\left(\mathrm{~m}^{3}\right)$ & Sample $3\left(\mathrm{~m}^{3}\right)$ \\
\hline Terra Modeler & $5,118,595.20$ & $4,749,489.20$ & $2,070,011.50$ \\
\hline Manual volume calculation & $5,114,139.41$ & $4,757,179.55$ & $2,069,697.67$ \\
\hline $\begin{array}{l}\text { Difference between manual } \\
\text { calculation and TerraModeler }\end{array}$ & $4,455.79$ & $7,690.35$ & 313.83 \\
\hline $\begin{array}{l}\text { Difference } \% \text { (all less than 1\% } \\
\text { difference) }\end{array}$ & 0.087 & 0.162 & 0.015 \\
\hline
\end{tabular}

This study shows that the LiDAR data has a great potential for volume calculation. Unlike other remote sensing techniques, LiDAR remote sensing technology is unique in the field of topography change mapping as it is able to measure features under vegetation by scanning the target with laser pulses highly effectively and accurately [10-12]. In 2014 another set of LiDAR data was captured over the Belaga area for another project. Therefore, with the availability of two sets of LiDAR and imagery data, captured on 12th February 2012 and 8th September 2014, for proposed hydropower dam development project in Sarawak, Malaysia, we are able to extract more information from them. Fig. 13 below shows the overlap areas of two projects in Belaga, collected in 2012 and 2014. This study area mostly comprises of dense jungle. 


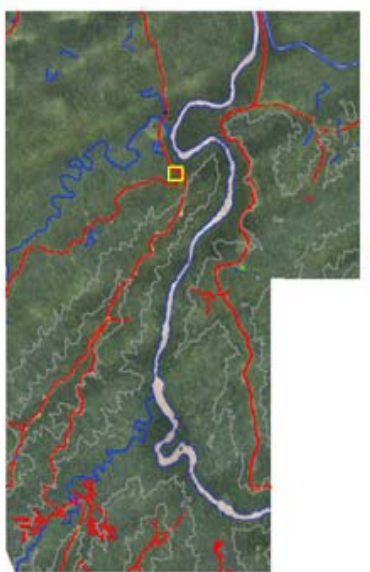

a)

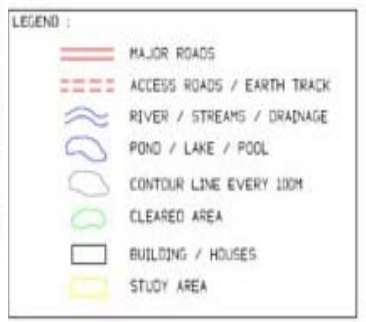

b)

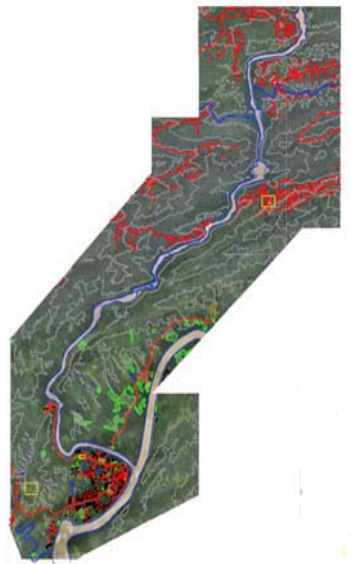

Fig. 13. Overlap area which depicts parts of the a) Northern b) Southern section of Belaga

To make sure that the changes in datasets is due to topographical effect and not processing procedure, cross sections of point clouds were used in built-up areas to validate the datasets. Static structures were used for the verification process. Fig. 14 represents the matching cross section in both datasets. 2012 and 2014 point clouds are represented as red and blue, respectively. The verification approves the unchanged status of the point clouds within two years.

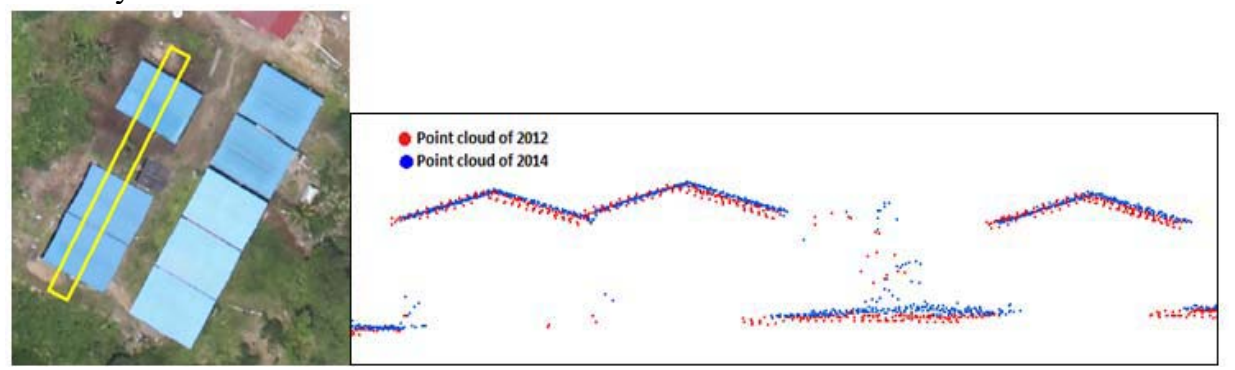

Fig. 14. Cross section of buildings in the study area and point cloud of the buildings and surrounding ground for 2012 in red and 2014 in blue.

\subsubsection{Comparison of area elevation changes}

Orthophoto images and DEM for 2012 and 2014 are shown in fig 15. visual inspection shows significant changes in the DEM surface during these two years.
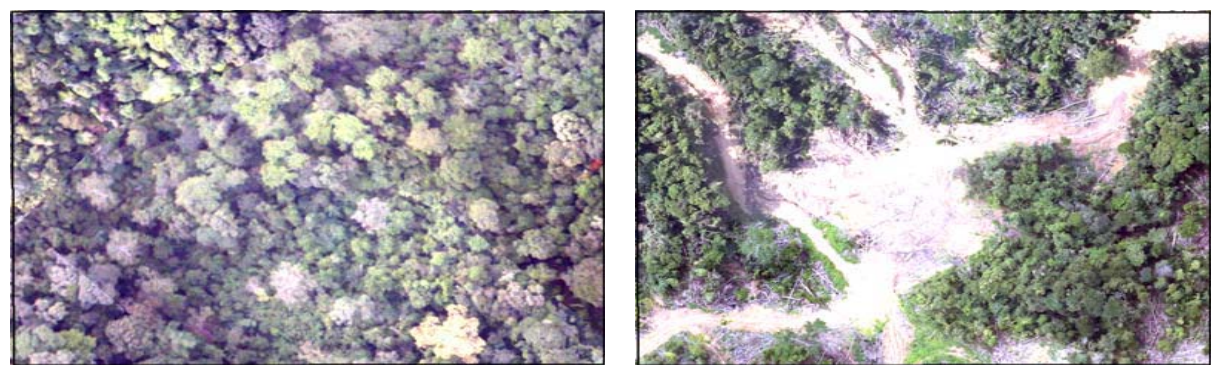
a)

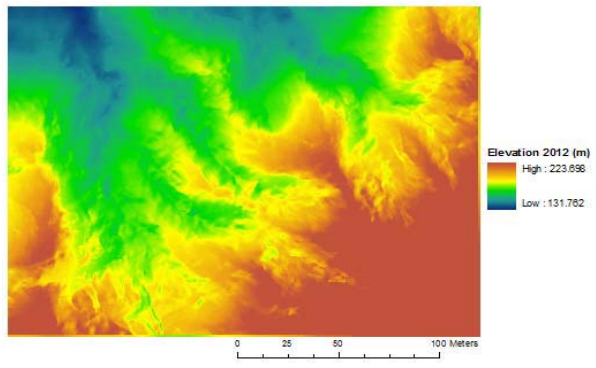

c) b)

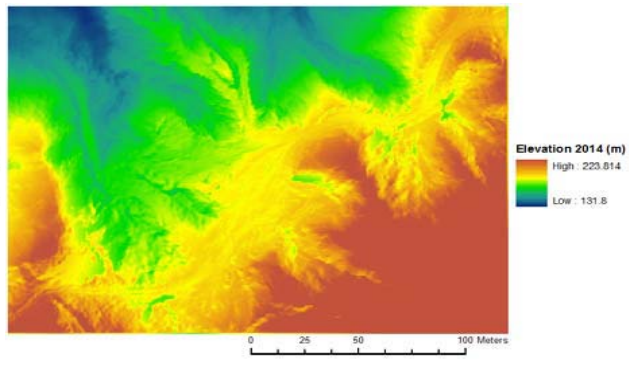

d)

Fig. 15. Study area for elevation changes, with the DEM generated from the LiDAR data. a) Orthophoto image in 2012, b) Orthophoto image in 2014, c) DEM of 2012 data and d) DEM of 2014 data.

Most of the changes are due to earthworks activities causing movement, compaction and expansion of soil. One main reason would be soil erosion. By monitoring changes in the terrain, the earthworks activities could be analysed. A more efficient method to identify areas that have been significantly cut and filled is analysing the raster data. This analysis is based on changes in the surface elevation during the two years period (2012 to 2014), which will identify the areas that have been affected by track construction, such that there is surface material loss, compaction, gain or expansion. Comparing the difference between the 2012 and 2014 raster grids shows the elevation changes represented in fig 16. The areas in blue indicate significant soil gain or expansion between 1 to $5.72 \mathrm{~m}$. The areas in red and orange indicate significant soil loss or compaction between -1 to $-6.07 \mathrm{~m}$. The green and yellow areas show little elevation change and cover approximately 60 percent of the study area.

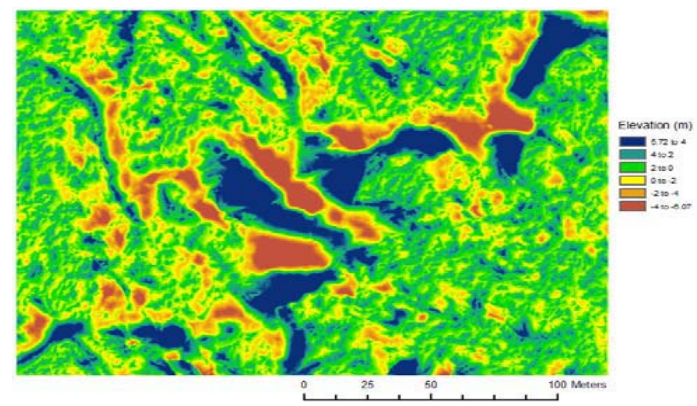

Fig. 16. Interpolated surfaces of area elevation changes.

\subsubsection{LiDAR for earthwork monitoring}

LiDAR technology provides very precise elevation data in detailed and clear imagery which allows for identification of features and vegetation. Areas expected to undergo elevation changes could be monitored easily by this data. Cross sections were used to analyse the changes using point cloud and visualization of the orthophoto mosaic images, as shown in fig 17.

Fig 17a shows the area underwent construction during the data acquisition in 2012; while in fig $17 \mathrm{~b}$, shows the road construction seems to be complete. Fig $17 \mathrm{c}$ presents the cross section of the road using both sets of data, which indicates that in two years, terrain 
changes have clearly occurred. In particular, there has been excavation work to lower ground levels by approximately $3 \mathrm{~m}$ for the road section.

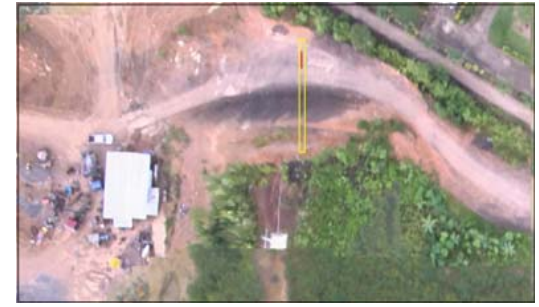

a)

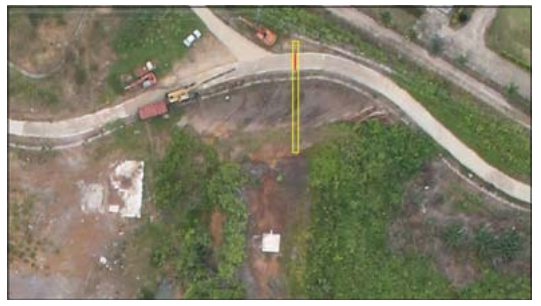

b)

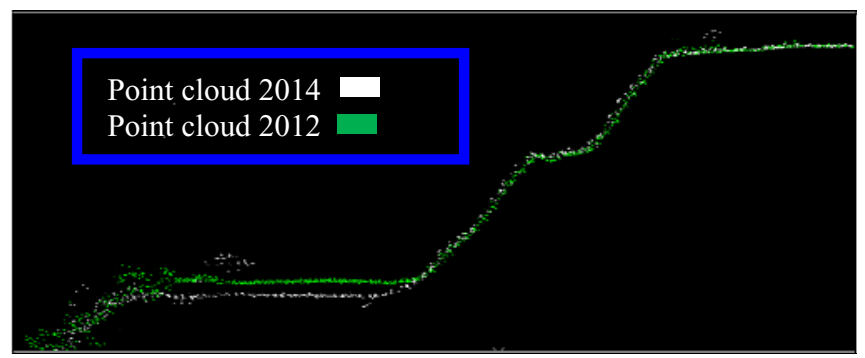

c)

Fig. 17. Images of road construction a) 2012, b) 2014, c) Cross section of point cloud in 2012 (green) and 2014 (white).

\subsubsection{Earthwork calculation}

In planning and design, LiDAR derived DEM and digital imagery is necessary to identify suitable alignments, evaluate alternative routes and create final design to optimize alignments and grades for the selected alternative. In fig. 16 the elevations of the same area for both years can be seen. There were minor changes in the elevation between 3.5 to -3.5 $\mathrm{m}$ as represented in fig. $18 \mathrm{c}$ ).

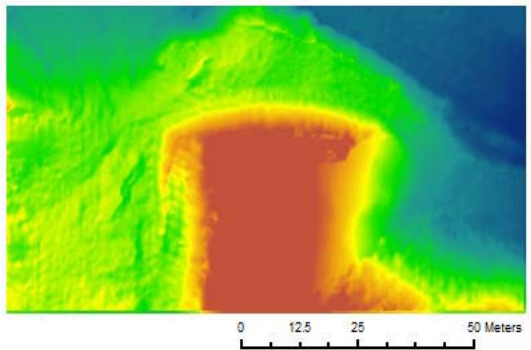

a)

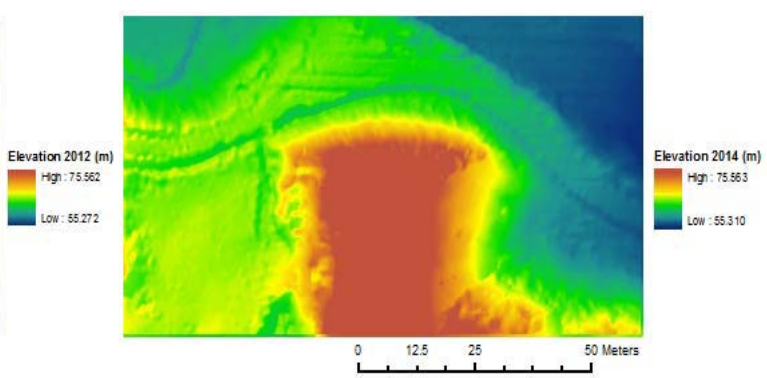

b) 


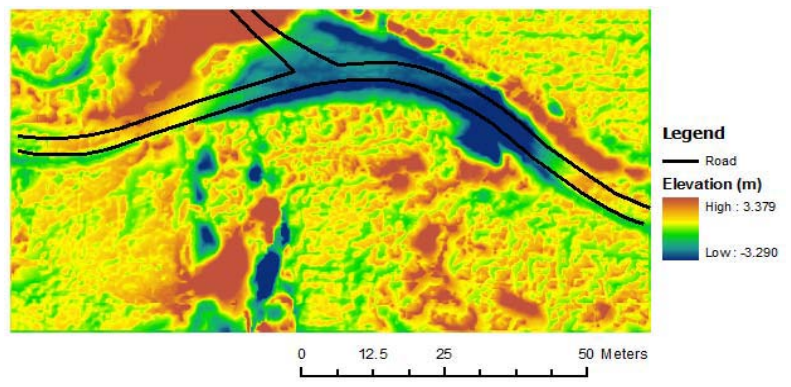

c)

Fig. 18. Elevation changes a) Elevation during road construction in 2012, b) Elevation in 2014 c) Elevation changes over 2 years

The cut and fill that is required for the desired area can be estimated using ArcGIS software. An estimated $120 \mathrm{~m}$ of the road segment undergoes cut and fill in this area. The road segment was divided into 12 sections of road, $3 \mathrm{~m}$ wide by $10 \mathrm{~m}$ long (Fig. 19). The following Table 2. demonstrates the estimate of cut and fill for each section.

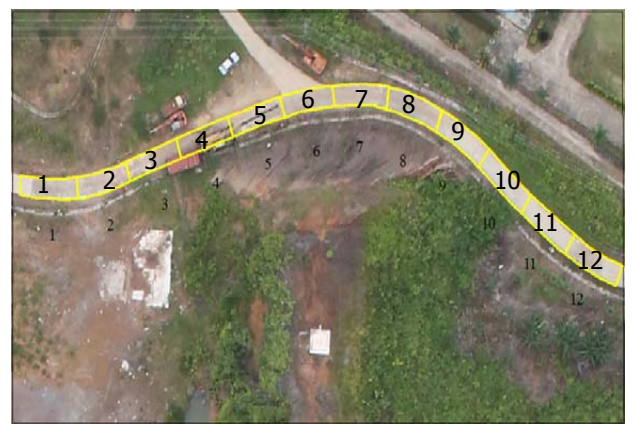

Fig. 19. 12 Sections of road

Table 2. Estimate of cut and fill

\begin{tabular}{|c|c|c|c|}
\hline \multirow{2}{*}{ Section } & \multicolumn{3}{|c|}{ Soil Volume $\left.\mathbf{( m}^{\mathbf{3}}\right)$} \\
\cline { 2 - 4 } & Cut & Fill & $\begin{array}{c}\text { Total material } \\
\text { disposed }\end{array}$ \\
\hline $\mathbf{1}$ & 0.9726 & 1.5848 & -0.6122 \\
\hline $\mathbf{2}$ & 0.0000 & 10.3208 & -10.3208 \\
\hline $\mathbf{3}$ & 0.0000 & 17.3907 & -17.3907 \\
\hline $\mathbf{4}$ & 4.9899 & 4.9836 & 0.0063 \\
\hline $\mathbf{5}$ & 21.5851 & 0.0000 & 21.5851 \\
\hline $\mathbf{6}$ & 27.3640 & 0.0000 & 27.3640 \\
\hline $\mathbf{7}$ & 24.5926 & 0.0000 & 24.5926 \\
\hline $\mathbf{8}$ & 28.4032 & 0.0000 & 28.4032 \\
\hline $\mathbf{9}$ & 31.8958 & 0.0000 & 31.8958 \\
\hline $\mathbf{1 0}$ & 28.3945 & 0.0000 & 28.3945 \\
\hline $\mathbf{1 1}$ & 4.8144 & 9.6253 & -4.8109 \\
\hline $\mathbf{1 2}$ & 0.1337 & 3.3214 & -3.1877 \\
\hline Total & 173.1458 & 47.2266 & 125.9192 \\
\hline
\end{tabular}


For this $120 \mathrm{~m}$ road section, a total of $125.9192 \mathrm{~m}^{3}$ of soil mass is estimated to be disposed. ArcGIS software has been used for these analyses and experience shows that more efficiency and accuracy could be gained using the LiDAR data for the purpose of the analysis.

\section{Result and discussion}

Three case studies demonstrate the roles of airborne LiDAR in different applications. In the first case study the results show how the airborne LiDAR data helped road engineers for detailed analysis of multiple alignments and helped them to select the best alignment while minimizing the cost and time of the project. In the second case study, the results showed how LiDAR data and assist transmission line engineers to model the construction costs of several alignment options effectively and efficiently compared to the conventional methods. The third case shows LiDAR data maximizes topological accuracy and volume measurements while minimizing survey time and costs. It is a fact that LiDAR data helps civil engineers in different ways in terms of fast data collection, accuracy, cost effectiveness, etc. As discussed previously, a single set of LiDAR data can be used for multiple users and purposes in different projects. Digital LiDAR informatin has a great potential to overcome the limitations of conventional methods beside cost and expenses as LiDAR data facilitate storing, sharing and visualization of data.

\section{Conclusions}

Airborne LiDAR survey technology has proven that it is one of the best methods to capture accurate information in three dimensions from the earth surface which provides maximum density of topographical \& digital imagery data for various industries, including engineering, infrastructure, environment, disaster and natural resources. This technology facilitates processing and analyzing a single dataset for multi-purpose applications such as pipeline, railway, road, transmission line, dam, telecommunications and urban planning, catchment and flood management while consuming minimum time, effort and expenses necessary throughout the project. Furthermore, engineering software such as MOSS (MXROAD) software with LiDAR data allows for detailed analysis for multiple alignments such that construction cost, time and environmental and social impact can be minimized in alignment design and earthworks volume calculation. Similarly, integration of PLS-CADD software with LiDAR data will provide significant information for transmission line engineers in terms of vegetation management, construction and maintenance, system operation and engineering. Besides the advantages of single LiDAR dataset, using multitemporal source of LiDAR data provides valuable information for various applications. Regarding the advantages of LiDAR technology, many companies are turning towards utilizing this technology for design, feasibility study, operation, maintenance, etc. 


\section{References}

1. J. Ramos, L. Marrufo, F.J. Gonzlez, Advances in Geoscience and Remote Sensing. InTech, ( 2009)

2. N. Kokkas, Customer presentation at the 2005 GXP regional user conference. Cambridge, England (2005).

3. M.L. Lu, and Z. Kieloch, IEEEE Transactions on Power Delivery ,23,3, (2008 )

4. R.V. Ussyshkin, L. Theriault, M. Sitar, and T. Kou, In Multi-Platform/MultiSensor Remote Sensing and Mapping (M2RSM),International Workshop on, 1-5. IEEE (2011).

5. X. Liu, J. Peterson, and Z. Zhang, Proceedings of the International Congress on Modelling and Simulation (MODSIM05). Modelling and Simulation Society of Australia and New Zealand Inc, (2005)

6. W. Uddin, ASCE Journal of Construction Engineering and Management, 134, 411, (2008)

7. T. Mccarthy, S. Fotheringham, M. Charlton, A. Winstanley, and V. O'Malley, Paper presented at The 5th International Symposium on Mobile Mapping Technologies. (2007)

8. T.R. Ganedra, Z. Zakaria, Malaysian Road Conference, 7th, Kuala Lumpur,(2007)

9. Z. Li, 'C. Zhu $C$. Gold, Digital Terrain Modelling: principles and methódology. CRC Press (2004)

10. K.H. Hsiao, J.K. Liu, M.F. Yu, and T.H. Tseng, XXth ISPRS Congress 7(5) (2004)

11. X. Yang (Ed.). Remote Sensing and Geospatial Technologies for Coastal Ecosystem Assessment and Management, Springer Science \& Business Media, (2008)

12. K. Lim, P. Treitz, M. Wulder, B. St-Onge and M. Flood, Progress in Physical Geography, 27,1. 88-106(2003) 